\title{
Creating Diamond-Like-Carbon (DLC) Templates Using Atomic Force Microscopy
}

\author{
Gregory S. Watson *, Sverre Myhra ${ }^{\dagger}$ and Jolanta A. Watson ${ }^{\ddagger}$ \\ *School of Science, Nanoscale Science and Technology Centre \\ Griffith University, Kessels Road, Nathan QLD 4111, Australia \\ Email: g.watson@griffith.edu.au \\ Telephone: (617) 3735-7531, Fax: (617) 3735-7656 \\ ${ }^{\dagger}$ School of Science, Nanoscale Science and Technology Centre \\ Email: Sverre.myhra@materials.ox.edu.au \\ ‡ Nanoscale Science and Technology Centre \\ Griffith University, Kessels Road, Nathan QLD 4111, Australia \\ Email: jolanta.watson@griffith.edu.au
}

\begin{abstract}
Pattern generation on the micro- and nano-scale is a vital ingredient in a vast variety of applications including biosensors and array devices. Current technologies are generally macroscopically-based, however a considerable increase in demand upon down-sizing must result in enabling meso/nanoscopic manipulation. Soft lithography is now routinely utilised in various applications with particular attention and importance placed on pattern transfer onto polymeric materials via the creation of masters. These are now fabricated using a number of techniques, all capable of producing well-defined surface topographies. The microelectronic industry utilises methods such as photolithography in order to fabricate such master templates at the micron scale.
\end{abstract}

Various polymers can be used to transfer patterns One of the most widely used is polydimethylsiloxane (PDMS). The elastomer is chemically resistant, has a low surface energy and readily conforms to different surface topographies. Obtaining a master is the limiting factor in the production of PDMS replicas.

The use of Diamond-Like-Carbon (DLC) as a master template is demonstrated in this study. PDMS micro/nano stamps and 3 dimensional PDMS structures have been created and routinely reproduced. Intricate surface relief patterns are formed on the DLC surface from lithographic techniques by Atomic Force Microscopy (AFM) operated in the electrical conductivity mode. The mode induces thermal oxidation on a DLC surface creating patterns in the tens of $\mathbf{n m}$. An example of a lithographic pattern created on a DLC surface by this method is shown in the topographical image in Fig. 1 (a). The template shown in Fig. 1 (a) was then coated with a layer of PDMS and thus used as a master. The resultant fabricated PDMS stamp is shown in Fig. 1 (b). The relief of the stamp correlates very well with the dimensions of the DLC master.
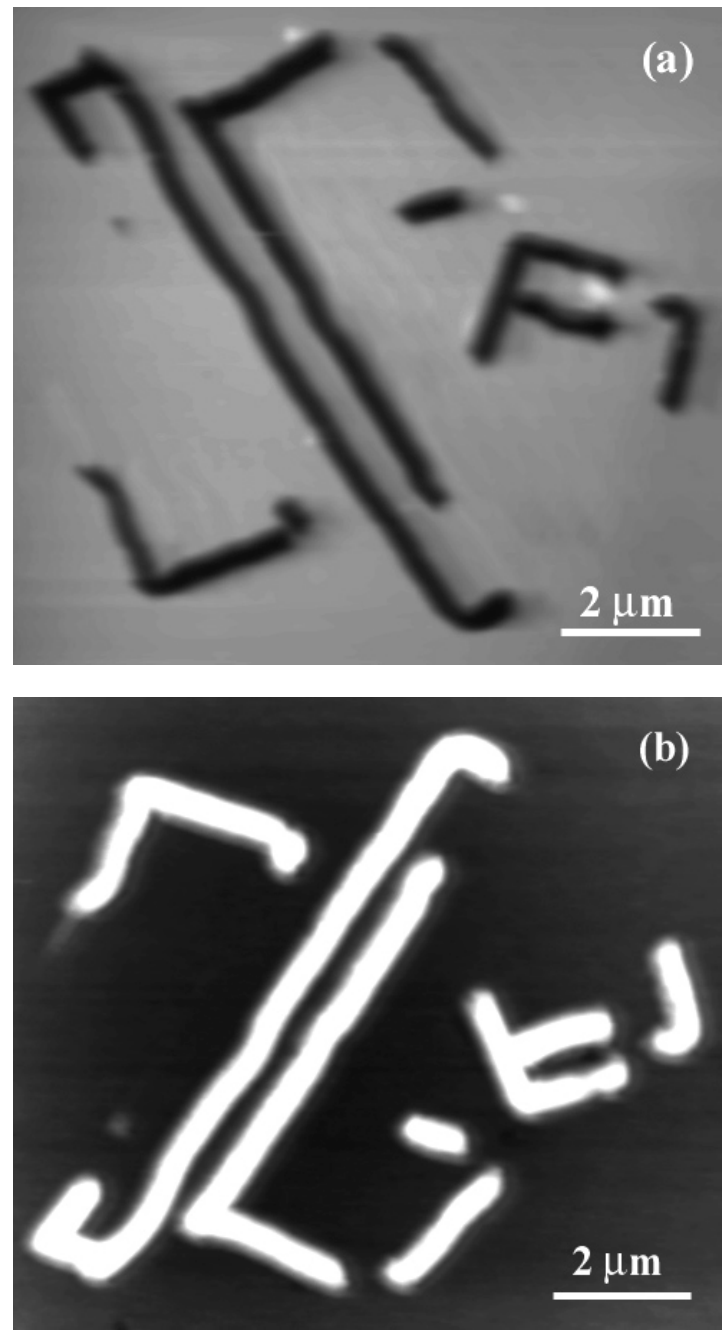

Figure 1. (a) DLC Template formed by tip-induced thermal oxidation. (b) The resultant PDMS structure - the stamp is formed by exposure of the polymer to the template topography. 
The DLC substrate is extremely durable, with the intricate details produced having a slope only limited by the aspect ratio of the tip ( $<10^{\circ}$ in this case). Attributes of the technique include:

- Features with line widths less than $20 \mathrm{~nm}$ can be formed on the DLC.

- The radius of curvature at edges can be less than $10 \mathbf{~ n m}$.

- Highly complex shapes can be fashioned.

- Feature depth can be controlled by DLC film thickness and/or by the bias voltage applied.

- The master is highly durable.

The master relief after patterning is extremely flat.

Keywords- Atomic force microscopy, PDMS, stamps, DLC, Diamond-like-Carbon.

\section{INTRODUCTION}

Various enabling Scanning probe Microscopy (SPM) based nano-technologies include nano-writing, nanolithography, surface patterning, templating, and nano-machining. Another set of methods known collectively as soft lithography is now being utilized for a large variety of applications including micromolding, microfluidic networks and microcontact printing [e.g., McDonald and Whitesides 2002]. The method utilizes the formation of stamps and elastomeric elements by exposing a polymer to a template. The templates are generally fabricated at the micron scale by well established lithographic techniques used in the microelectronic industry, such as photolithography. Creating templates on the nano-scale however, has been a limiting factor.

Diamond-Like-Carbon (DLC) is the generic term for a class of materials that can be synthesized by a variety of wellestablished routes, leading to phases that are diamond-like. The hardness and other mechanical properties are comparable to those of crystalline diamond.

The transference of a pattern from a master is now possible with the aid of various polymers, with polydimethylsiloxane (PDMS) being one of the most common. The polymer is attributed with a wide range of useful physical, mechanical and chemical properties including transparency, surface hydrophobicity, constant and high ductility over a wide range of temperatures, low toxicity, high electrical resistance, longterm stability and flexibility [Olah et al 2005]. The fabrication and use of micro/nano stamps and fluidic channels using PDMS has been demonstrated in various studies [e.g., Kane et al 1999, Hu et al 1999, Schueller et al 1999, Deng et al 2002].

We demonstrate the use of DLC as a template for producing PDMS micro/nano stamps and 3-dimensional polymer structures by Atomic Force Microscopy (AFM) operated in the electrical conductivity mode.

\section{EXPERIMENTAL DETAILS}

\section{A. Specimen Materials}

Electrically conductive IB-DLC films were deposited onto polished n-type Si substrates. The process technology was an ion beam assisted variation of the generic chemical vapour deposition route. The substrate was maintained at $80^{\circ} \mathrm{C}$ at an ambient pressure of $10^{-6}$ Torr, while being exposed to a partial pressure of polyphenyl ether from a source held at $150^{\circ} \mathrm{C}$. The outcome was a wear-resistant diamond-like carbon film, although with considerable graphitic character, and exhibiting low friction (0.1-0.2 for contact loads up to $100 \mathrm{~N}$ ). Root Mean Square (RMS) surface roughness of the films was in the range $0.25-0.3 \mathrm{~nm}$ inferred from AFM imaging over fields of view of $1 \times 1$ and $2 \times 2 \mu \mathrm{m}^{2}$.

\section{B. SPM instrumentation}

The experiments and analysis were carried out with a JEOL JSTM-4200D and a JSPM-4200 multi-technique instrument. A typical sequence of surface modification and analysis proceeded with the acquisition of an image in the contact mode over a large field of view, typically 1-4 $\mu^{2}$ with a lever-imposed force loading of $10-100 \mathrm{nN}$ and zero bias, in order to ascertain that the chosen surface region was free of any artefacts. The surface modification at a point, or over a small field of view, was then carried out, at particular values of bias voltage, scan speed and force loading. A larger field of view, centred on the location of the surface modification, was then rescanned at zero bias in order to reveal the topographical effects induced by the tip-to-substrate bias. The force-loading was maintained constant throughout the runs, whenever appropriate, as were the parameters used for topographical analysis, in order to improve reproducibility of outcomes.

\section{Probes}

The probe consists of a lever with an integral conical tip attached at its free end. The tip-to-surface point of contact defines the interaction volume, whereby a large amount of information such as topography, strength of in-plane and outof-plane forces is extracted. The point of contact is also that through which purposeful manipulation is effected.

Beam-shaped levers were used in order to ensure that only simple bending modes needed to be considered. The data for the length, width and thickness of the lever were $350 \mu \mathrm{m}, 35$ $\mu \mathrm{m}$ and $2 \mu \mathrm{m}$, respectively. The radius of curvature at the tip apex, $R_{\text {Tip }}$, aspect ratio (opening half angle) of the tip, $A_{r}$, and surface chemistry $\left(<10 \mathrm{~nm},<10^{\circ}\right.$ and native Si-oxide film, respectively) were obtained from the suppliers' specifications.

The tips utilized for DLC manipulation were also characterized by 'reverse' imaging over a grid of spikey features; the radii of curvature were consistent with those claimed by the manufacturer. The probes were also subjected to I-V characterization before and during experimental runs by investigation of point-contact resistance on a Si-substrate covered by a gold film. Those probes that exhibited contact resistance greater than $10^{10} \Omega$ were discarded.

Many polymers can be considered as 'soft' objects; therefore the imposition of forces at the point of contact will cause deformation and indentation, and will lead to an increase in contact area. Thus it is necessary to use levers with force constants, $k_{N}$, $\leq 0.1 \mathrm{Nm}^{-1}$ in order to improve resolution and avoid unintended surface modification. Levers with force constants $\geq 4 \mathrm{Nm}^{-1}$ result in considerable undesired surface manipulation. 


\section{RESULTS AND DiSCUSSION}

\section{A. Thermal Oxidation on DLC}

Well defined 3-dimensional lithographic patterns can be produced via nano-manipulation by tip-induced local prompt oxidation of electrically conductive DLC material [Myhra and Watson 2005]. The extent of spatial resolution for 3dimensional structures is illustrated in the image and contour line in Fig. 2 (a) and (b), respectively. The square pit shown in Fig. 2 (a) was obtained by scanning at a sample bias of $4.5 \mathrm{~V}$ for a total duration of $90 \mathrm{~s}(1.5 \mathrm{~ms}$ per pixel over a field of view made up of $256 \times 256$ pixels). The excavated area depth is $100 \times 100 \mathrm{~nm}^{2}$ and $12-13 \mathrm{~nm}$, respectively. The side-wall slope and shape of the excavated pit was consistent with the tip apex being described by a parabolic section. The bottom of the pit was flat to within $\pm 1 \mathrm{~nm}$. It is noteworthy that the 'roughness' of a line drawn along an edge of the pit was less than $5 \mathrm{~nm}$. The in-plane curvature at a corner and the out-of plane curvature at an edge were both ca. $5 \mathrm{~nm}$.

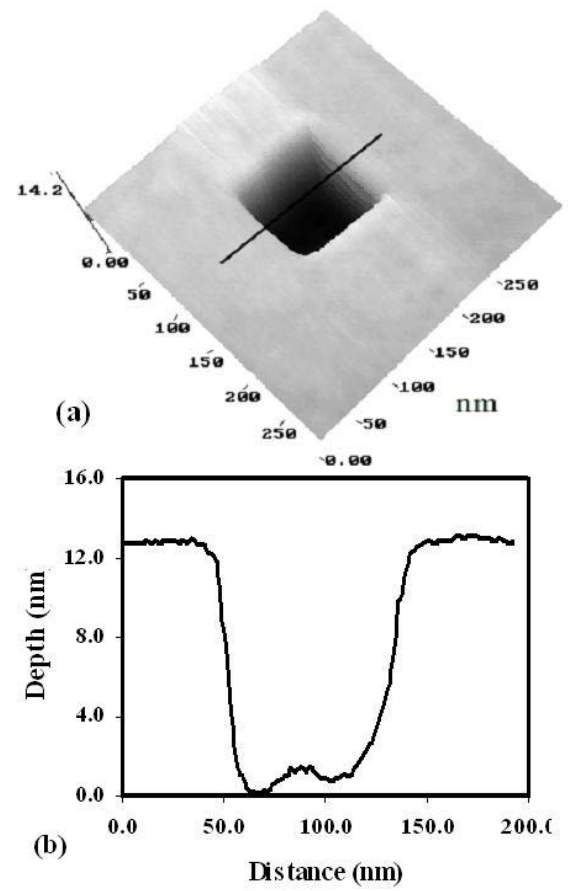

Figure 2. Contact mode image as a 3-dimensional representation in (a) and a cross-sectional contour line in (b). The outcome illustrated is a result of carrying out tip-induced oxidation in air. A positive sample bias of $4.5 \mathrm{~V}$ was applied for $90 \mathrm{~s}$, with the tip being scanned over a $100 \times 100 \mathrm{~nm}^{2}$ field of view composed of $256 \times 256$ pixels.

A series of trenches, or channels, were each inscribed by a single linear raster of the tip. The channels were generated by a dwell-time of $10 \mathrm{~ms}$ per pixel for a total of 128 pixels per line. The channels were created $500 \mathrm{~nm}$ in length and offset by $100 \mathrm{~nm}$. A 3-dimensional image and a transverse contour line are shown in Fig. 3 (a) and (b), respectively. The dependence on the tip-to-sample bias was explored over a range of 5.0-6.5 $\mathrm{V}$ at $0.5 \mathrm{~V}$ increments. The full width at half-maximum depth was in the range $20-40 \mathrm{~nm}$. The scan direction was from topleft to bottom-right. The application of bias was synchronized with the start of the line scan. Accordingly the irregularities at the beginning of the line (seen at the top of Fig. 3 (a)) are indicative of the time delay for establishing stable (thermal) conditions.
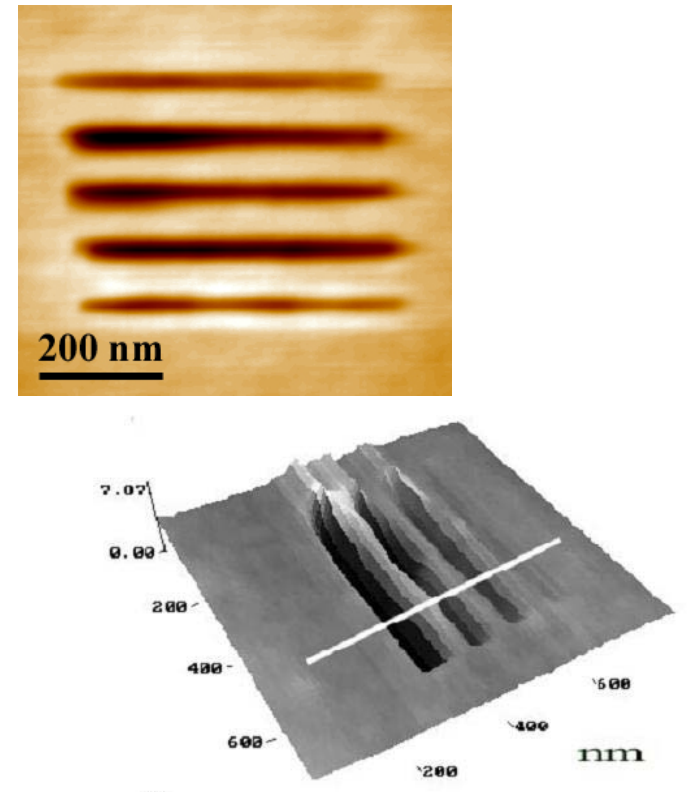

(a)

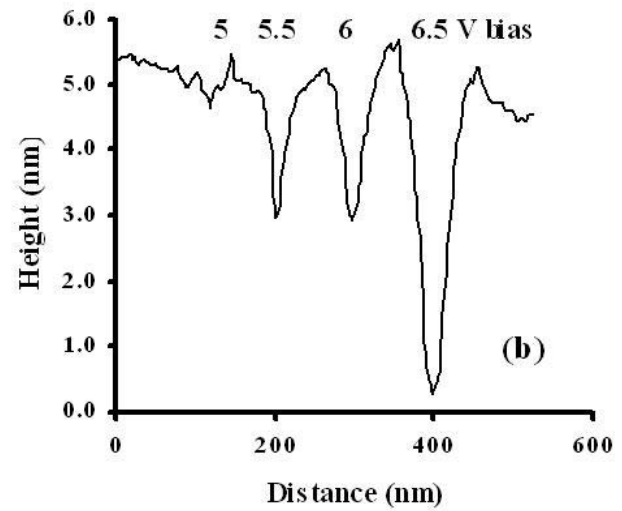

Figure 3. Contact mode image as a 3 dimensional representation (a) and contour lines of trenches (b) inscribed at bias voltages from 5 to $6.5 \mathrm{~V}$. Each line was generated by 128 pixels and a dwell-time of $10 \mathrm{~ms} /$ pixel.

\section{B. DLC template and PDMS stamp formation}

As seen in Fig. 2 and Fig. 3 above, intricate details can be produced on a DLC surface by inducing thermal oxidation. The formation of features in the tens of nm have now been routinely reproduced. Figure 6 shows a lithographic pattern created by thermal oxidation. The DLC substrate is extremely durable, with the intricate details produced having a slope only limited by the aspect ratio of the tip ( $<10^{\circ}$ in this case).

The template created in figure 6 was coated with a layer of PDMS and thus used as a master. The PDMS was subsequently peeled off the DLC surface and imaged with a soft lever in order to avoid manipulation. The resultant fabricated PDMS stamp is shown in figure 7 . The relief of the stamp correlates very well with the dimensions of the DLC master. 
A 3 dimensional image of the PDMS stamp, and corresponding line profile along one of the longer stamp elements (A), are shown in Fig. 3 (a) and (b), respectively. The line profile perpendicular to one of the shorter elements (B), as shown in (a), for the PDMS stamp and the original DLC template are shown in Fig. 3 (c) and (d) The figure illustrates the nm scale surface roughness and height of the stamp (ca. $250 \mathrm{~nm}$ ) with (c) and (d) showing comparable dimensions of the master and resultant stamp.
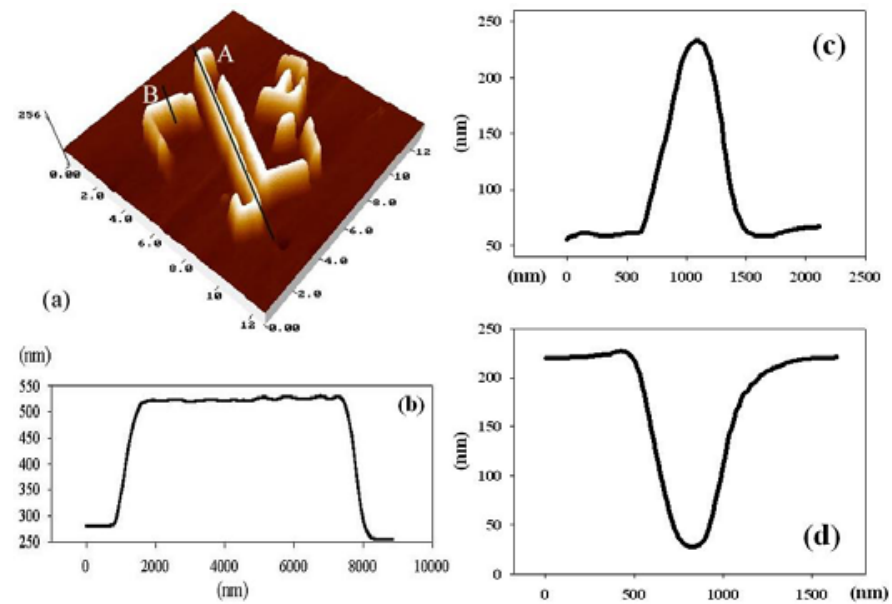

Figure 4. (a) 3 dimensional representation of the stamp shown in figure 7 revealing more height detail. (b) shows a corresponding line profile showing the surface roughness of the stamp along one of the longer elements (A). (c) A line profile perpendicular to one of the shorter arms (B). (d) is a similar line profile but taken on the original DLC master. The dimensions are comparable.

\section{Abbreviations and Acronyms}

\section{CONCLUSION}

In this study we have demonstrated the use of DiamondLike-Carbon (DLC) as a master template for producing polymer micro/nano stamps and 3-D polymer structures. Intricate surface relief patterns can be formed on the DLC surface from lithographic techniques by Atomic Force Microscopy (AFM) operated in the electrical conductivity mode.

Attributes of the technique as a template include
- Features with line widths less than $20 \mathrm{~nm}$ can be formed on the DLC.

- The radius of curvature at edges can be less than 10 nm.

- The slope of the features can only be limited by the aspect ratio of the tip.

- Highly complex shapes can be fashioned.

- Feature depth can be controlled by DLC film thickness and/or by the bias voltage applied.

- The master is highly durable.

The master relief after patterning is extremely flat.After the text edit has been completed, the paper is ready for the template. Duplicate the template file by using the Save As command, and use the naming convention prescribed by your conference for the name of your paper. In this newly created file, highlight all of the contents and import your prepared text file. You are now ready to style your paper; use the scroll down window on the left of the MS Word Formatting toolbar.

\section{ACKNOWLEDGMENT}

The work was funded in part by a grant from the Australian Research Council.

\section{REFERENCES}

[1] G. Eason, B. Noble, and I. N. Sneddon, "On certain integrals of Lipschitz-Hankel type involving products of Bessel functions," Phil. Trans. Roy. Soc. London, vol. A247, pp. 529-551, April 1955. (references)

[2] J. Clerk Maxwell, A Treatise on Electricity and Magnetism, 3rd ed., vol. 2. Oxford: Clarendon, 1892, pp.68-73.

[3] I. S. Jacobs and C. P. Bean, "Fine particles, thin films and exchange anisotropy,” in Magnetism, vol. III, G. T. Rado and H. Suhl, Eds. New York: Academic, 1963, pp. 271-350.

[4] K. Elissa, "Title of paper if known,” unpublished.

[5] R. Nicole, “Title of paper with only first word capitalized,” J. Name Stand. Abbrev., in press.

[6] Y. Yorozu, M. Hirano, K. Oka, and Y. Tagawa, "Electron spectroscopy studies on magneto-optical media and plastic substrate interface,” IEEE Transl. J. Magn. Japan, vol. 2, pp. 740-741, August 1987 [Digests 9th Annual Conf. Magnetics Japan, p. 301, 1982].

[7] M. Young, The Technical Writer's Handbook. Mill Valley, CA: University Science, 1989. 